\title{
Mineração de Dados Educacionais: uma análise sobre o engajamento de usuários em mundos virtuais
}

\author{
Fabrício Herpich ${ }^{1}$, Felipe Becker Nunes ${ }^{2}$, Sílvio César Cazella ${ }^{1,2}$ e \\ Liane Margarida Rockenbach Tarouco ${ }^{1}$ \\ ${ }^{1}$ Universidade Federal do Rio Grande do Sul (UFRGS) \\ Av. Paulo Gama, 110, Porto Alegre, RS, Brasil - CEP 90040-060 \\ ${ }^{2}$ Universidade Federal de Ciências da Saúde de Porto Alegre (UFCSPA) \\ Rua Sarmento Leite, 245, Porto Alegre, RS, Brasil - CEP 90050-170 \\ \{fabricio.herpich, nunesfb, silvio.cazella\}@gmail.com, \\ liane@penta.ufrgs.br
}

\begin{abstract}
This article describes an investigation conducted in order to identify the aspects related to the engagement of users in virtual worlds applied to education. To accomplish this, the validation of a physical virtual lab with ten students of computer courses is presented. After these users navigate in the laboratory, the mining techniques of educational data was performed through the association task, to show the patterns of engaged users, as well as the elements that made possible the involvement of the same with the environment. Through these results, also was possible to establish when the users has not reached a certain level of involvement with the virtual environment, enabling the implementation of corrective actions to the rescue of these users.
\end{abstract}

Resumo. Este artigo descreve uma investigação conduzida com o objetivo de identificar os aspectos relacionados ao engajamento de usuários em mundos virtuais aplicados na educação. Para alcançar este objetivo, é apresentada a validação de um laboratório virtual de física com dez estudantes de cursos da computação. Após a navegação destes usuários, são desempenhadas as técnicas de mineração de dados educacionais, através da tarefa de associação, para evidenciar os padrões dos usuários engajados, bem como os elementos que possibilitaram o envolvimento dos mesmos com o ambiente. Por meio destes resultados, também foi possivel estabelecer quando os usuários não alcançaram um determinado nível de envolvimento com o ambiente, viabilizando a execução de ações corretivas para o resgate destes usuários.

\section{Introdução}

A expansão das pesquisas envolvendo o uso das Tecnologias da Informação e Comunicação no âmbito educacional, tem resultado em diversas iniciativas que buscam auxiliar os professores e alunos nos processos de ensino e aprendizagem. Os mundos virtuais estão presentes neste contexto, em que de acordo com Orgaz et al. (2012), tais ambientes tem como objetivo disponibilizar espaços 3D onde o estudante pode transitar e vivenciar experiências em um ambiente altamente interativo. 
$\mathrm{O}$ aspecto interdisciplinar se torna evidente ao utilizar este ambiente com foco na área educacional, visto que as demais áreas também podem ser incluídas nos processos, como química, física, engenharia, entre outras. O potencial existente nos mundos virtuais para a construção de simulações de fenômenos e a interação ativa com estes experimentos, agregado à diversidade de recursos multimídias que podem ser explorados no ambiente, o torna uma alternativa instigadora para auxiliar no processo de aprendizagem dos alunos.

Neste contexto, o mundo virtual possui como características o alto nível de interação e aprendizagem ativa, colaboração e comunicação entre os participantes, buscando fornecer uma sensação de imersão ao usuário, para que o mesmo sinta-se engajado nas atividades a serem desempenhadas no ambiente. Desta forma, o envolvimento do usuário se torna um ponto importante a ser analisado durante sua interação com ambientes educacionais, em que O'Brien e Toms (2008) consideram que tal fator depende da profundidade de participação que os utilizadores são capazes de alcançar, em relação a cada atributo experimental disponível no sistema.

Para desempenhar tal análise, torna-se necessário efetuar a coleta de dados resultantes das interações do usuário com o ambiente, como a assiduidade de acesso, locais que visitou e quanto tempo permaneceu, bem como as ações que executou, sua interação com os demais usuários no ambiente. Para efetuar tal estudo, se abre uma nova gama de possibilidades por intermédio do uso de técnicas de Mineração de Dados Educacionais (MDE). Segundo Romero e Ventura (2010), a área de MDE está preocupada com o desenvolvimento, pesquisa e aplicação de métodos computadorizados para detectar padrões em grandes conjuntos de dados educacionais, que de outra forma seriam difíceis ou impossíveis de analisar devido ao enorme volume de dados.

A fim de examinar esta dimensão em específico, este artigo tem como objetivo desempenhar uma análise dos dados coletados em meio a interação de dez usuários, com o intuito de estabelecer quais elementos e recursos educacionais encontrados no laboratório virtual são capazes de promover o engajamento dos mesmos, assim como possibilitar a aprendizagem sobre o conteúdo. Para a coleta das informações referentes a interação dos usuários, foi construído um laboratório virtual de física no mundo virtual OpenSimulator e desenvolvido um experimento na área de física, sobre a bobina de Nikola Tesla, sendo que por meio das interações dos usuários neste laboratório, com o experimento, recursos educacionais e atividades sobre o conteúdo apresentado, foram coletadas as informações utilizadas na execução da tarefa de mineração de dados.

O artigo está estruturado em seções descritas a seguir: a seção 2 apresenta os fundamentos teóricos sobre mundos virtuais e os conceitos relacionados ao engajamento de usuários. A seção 3 apresenta uma introdução sobre a área de mineração de dados educacionais e a técnica de regras de associação utilizada para a análise dos dados deste estudo. Na seção 4 são apresentados os trabalhos relacionados. A seção 5 apresenta os procedimentos metodológicos adotados para a execução desta investigação. Ao longo da seção 6 é apresentado o experimento utilizado para a coleta de dados, já na seção 7 são descritas as regras de associação descobertas durante o processo de mineração de dados e a análise dos conhecimentos evidenciados. A seção 8 apresenta as conclusões e perspectivas futuras de trabalho. 


\section{Mundos Virtuais}

Griol et al. (2014) tem o entendimento de que os mundos virtuais podem ser considerados ambientes gráficos simulados por computador, no qual os seres humanos convivem com outros usuários através de seus avatares. Ambientes como o Second Life, OpenSimulator (OpenSim) e OpenWonderland são exemplos de aplicações que possibilitam a criação de mundos virtuais.

A interação no ambiente ocorre por meio de uma aplicação denominada viewer, na qual o usuário coloca o endereço de acesso ao mundo virtual, suas credencias e efetua a conexão. Existem diferentes viewers que podem ser utilizados, como o Singularity, Imprudence e Firestorm, em que cada um destes possui características e limitações próprias. Conforme Nunes et al. (2014), a escolha de um viewer que não atenda aos interesses do usuário, pode acarretar em diversos problemas, como a visualização incorreta dos objetos importados, limitando sua interação e impedindo a realização de atividades. Portanto, deve-se verificar a aplicação que melhor se adéqua as necessidades de uso do ambiente e permite a visualização e interação com os objetos.

Com relação a aplicação de atividades envolvendo os mundos virtuais no âmbito educacional, se configura viável a possibilidade de criação de laboratórios virtuais que possibilitem a demonstração de simulações de experimentos, que em alguns casos são complexos de serem reproduzidos no mundo real. Tal procedimento proporciona que estes experimentos sejam realizados de forma virtual, o que diminui os custos com infraestrutura, materiais e pessoas envolvidas, além de remover os riscos de exposição dos alunos com experimentos perigosos.

Áreas como química, física, biologia e engenharia possuem um grande potencial a ser explorado nos mundos virtuais, visto que diversos tópicos de ensino podem ser trabalhados no ambiente. Dentro deste contexto, é importante ressaltar que a formulação de atividades didáticas no ambiente devem ser cuidadosamente planejadas, para que o usuário consiga interagir adequadamente e se sinta motivado a prosseguir utilizando o ambiente. A subseção a seguir descreve, de forma específica, os detalhes inerentes a esta questão, abordando detalhes sobre o engajamento dos usuários em mundos virtuais.

\subsection{Engajamento de Usuários em Mundos Virtuais}

Um tópico frequentemente abordado por engenheiros de software que trabalham com a implementação de aplicativos para os mais variados fins, consiste na questão de como estabelecer meios para alcançar o envolvimento dos usuários com as ferramentas em que os mesmos estão interagindo.

Em mundos virtuais, esta questão não só tem sido discutida, como tem gerado certa complexidade na implementação destes ambientes virtuais imersivos, em virtude destes ambientes já disponibilizarem elementos que por si só promovem o envolvimento dos seus usuários, pois possibilitam a interação com objetos tridimensionais, simulações, entre outras mídias. No entanto, por mais que sejam ambientes atrativos aos seus usuários, ainda são deficitários no aspecto de envolvê-los e cativá-los, promovendo o interesse dos mesmos em retornarem ao ambiente para continuarem as suas interações.

Diante desta perspectiva, é possível afirmar que o engajamento de usuários em mundos virtuais, configura-se em um assunto emergente, que busca congregar os 
conceitos já estabelecidos por autores da área de Interação Humano-Computador (IHC), com o desenvolvimento destes ambientes virtuais imersivos. Neste aspecto, Herpich e Tarouco (2016) corroboram afirmando que a área de IHC apresenta conceitos e princípios substanciais, do ponto de vista em oportunizar a aproximação dos ambientes voltados a educação aos anseios de seus usuários. O'Brien e Toms (2008) defendem que o engajamento dos usuários consiste nas experiências que os mesmos interagem, sendo que a qualidade destas experiências depende dos recursos estéticos inovadores e da usabilidade do sistema; da capacidade dos usuários em estarem presentes e envolveremse com as experiências; bem como da avaliação das experiências adquiridas.

\section{Mineração de Dados Educacionais}

A Mineração de Dados Educacionais pode ser considerada uma dimensão da mineração de dados, em virtude de empregar os métodos e procedimentos da área, mas com um objetivo final diferente, o qual consiste na análise de grandes volumes de dados à procura de padrões recorrentes que possam auxiliar na tomada de decisões relacionadas ao âmbito educacional, e.g. regras de associação, classificação e predição.

Neste sentido, Costa et al. (2013) afirmam que a MDE procura desenvolver ou adaptar métodos e algoritmos de mineração existentes, de tal modo que possibilitem compreender melhor os dados em contextos educacionais, produzidos principalmente por estudantes e professores. Já Baker, Isotani e Carvalho (2011) corroboram afirmando que a MDE possui como objetivo o desenvolvimento de métodos para explorar conjuntos de dados coletados em ambientes educacionais, tornando factível a compreensão dos alunos, bem como a forma e o contexto na qual a aprendizagem ocorre, além de outros fatores que influenciam a aprendizagem dos mesmos.

Para o desempenho deste estudo, foi utilizada a tarefa de regras de associação, a qual foi implementada sobre um conjunto de dados por meio do algoritmo Apriori. A regra de associação tem como intuito realizar a análise do conjunto de dados, a fim de encontrar relacionamentos entre uma variável e outra. Castanheira (2008) acrescenta que o objetivo das regras de associação é encontrar tendências que possam ser usadas para entender e explorar padrões de comportamento dos dados.

\section{Trabalhos Relacionados}

Com o intuito de fornecer uma visão mais clara de algumas pesquisas que estão sendo desenvolvidas dentro deste escopo, a seguir são descritos alguns trabalhos que envolvem o uso de mundos virtuais com técnicas de mineração de dados educacionais..

A pesquisa desenvolvida por Fernández-Gallego et al. (2013) buscou estudar a descoberta de fluxos de aprendizagem e verificar a sua conformidade através de técnicas de mineração de dados. Uma plataforma adaptada do OpenSim foi construída para realizar a coleta de informações resultantes de eventos realizados por alunos e professores. Os autores apresentaram a construção de um framework a ser seguido para obter tais informações e como as mesmas podem ser analisadas por meio de algoritmos de mineração de dados e Learning Analytics.

O estudo de Orgaz et al. (2012) apresenta uma análise de comportamento por meio das ações dos usuários, como movimentação, objetos que olharam e interagiram. Para compor a amostra de informações utilizadas nas análises, foi desenvolvido um 
mundo virtual com base no OpenSim e denominado de VirtUAM. Posteriormente, por meio do uso de técnicas de agrupamento (clusters), os dados coletados foram analisados com o intuito de encontrar possíveis padrões de comportamento em grupos. Os resultados obtidos demonstraram que o uso desta técnica é viável, pois revela um alto grau de precisão na classificação de padrões de comportamento dos usuários.

No trabalho de Nunes et al. (2015), um estudo acerca das interações dos usuários nos mundos virtuais foi realizado, com o intuito de verificar os possíveis padrões para usuários do OpenSimulator por meio do uso de mineração de dados. Os dados utilizados na análise foram gerados a partir da ferramenta GenerateData, de forma sintética, apenas para compor os atributos relacionados a interação do usuário no ambiente. Por meio do uso do algoritmo Apriori, foram observadas as regras de associação resultantes, demonstrando a viabilidade na constatação de padrões de interação nos usuários, sendo este um estudo inicial sem usuários reais.

Os estudos descritos nesta seção evidenciam a atualidade do tema e o diferencial desta pesquisa, consiste na análise do engajamento dos usuários no mundo virtual, através da tarefa de mineração de dados que possibilita estabelecer regras de associação e evidenciar os aspectos que promovem o envolvimento destes usuários.

\section{Procedimento Metodológico}

Para a realização desta pesquisa, foram realizados testes com dez usuários, os quais foram instruídos a interagirem com o laboratório virtual de física e seus recursos educacionais, tais como objetos 3D, vídeos e simulações. Por meio da interação destes usuários, foi possível constituir a base de dados com informações sobre a navegação dos mesmos, através do monitoramento das interações dos usuários, as quais foram armazenadas em uma base de dados por meio do uso de sensores no mundo virtual.

Findados os testes com os usuários e a coleta de informações que compõem a base de dados, utilizou-se o método conhecido como extração de conhecimento de base de dados, do inglês Knowledge-Discovery in Databases (KDD) (Fayyad, PiatetskyShapiro e Smyth, 1996), que estabelece as etapas para a aquisição de conhecimento ao longo do processo de mineração de dados, os quais são:

a) Seleção: durante a etapa de seleção, foi realizada uma análise no banco de dados, para estabelecer os conjuntos de dados que seriam utilizados para a presente pesquisa, a fim de destacar aqueles elementos que possuem maior relacionamento com o que está sendo proposta nesta mineração de dados.

b) Pré-Processamento: já na etapa de pré-processamento, ocorreu a organização e preparação dos dados, sendo realizado o tratamento e correção dos conjuntos de dados selecionados na etapa anterior, com o intuito de retificar informações ausentes ou inconsistentes na base de dados, para não comprometer a execução do algoritmo e por consequência, os conhecimentos extraídos ao final do processo.

c) Formatação: ao longo da etapa de formatação, os conjuntos de dados obtidos na etapa anterior, foram transformados, formatados e reorganizados em uma ordenação específica para a interpretação na etapa seguinte. 
d) Mineração de Dados: durante a etapa de mineração de dados, os conjuntos de dados selecionados e formatados nas etapas anteriores, foram importados para a ferramenta Weka, em que foi realizada a leitura destes dados e aplicado o algoritmo e selecionada a tarefa de mineração de dados.

e) Interpretação / Avaliação: nesta etapa foi realizada a avaliação dos resultados obtidos durante o desempenho do processo de mineração de dados executado, a fim de verificar se houve alguma ocorrência que estabeleceu um padrão, relacionamento e a descoberta de um novo conhecimento.

Para desempenhar o procedimento descrito acima, foi utilizada a ferramenta Weka, a qual congrega uma série de algoritmos prontos para serem executados e que possibilita a prática de diferentes tarefas de mineração de dados. Neste estudo, foi executada a tarefa de associação, através da qual é possível descobrir regras que evidenciam elementos, padrões e relacionamentos frequentes em um mesmo conjunto de dados.

\section{Experimento com a Bobina de Tesla}

Os usuários que participaram desta avaliação, foram instruídos a utilizar o laboratório virtual de física construído no OpenSim, o qual compõe o objeto de estudo analisado nesta pesquisa e tem como objetivo auxiliar os seus usuários durante o processo de aquisição dos conhecimentos de física vinculados aos experimentos de Nikola Tesla, desenvolvendo os conceitos relacionados a área de eletromagnetismo, através de experimentos 3D e simulações (Figura 1).

Para que fosse possível oferecer suporte ao processo de ensino e aprendizagem destes usuários, foram implementados diversos recursos educacionais junto ao laboratório virtual de física, como objetos tridimensionais, imagens, vídeos, bem como conteúdos interativos, tais como animações e simulações, com o intuito de exemplificar as leis da física com situações inerentes ao cotidiano dos mesmos.

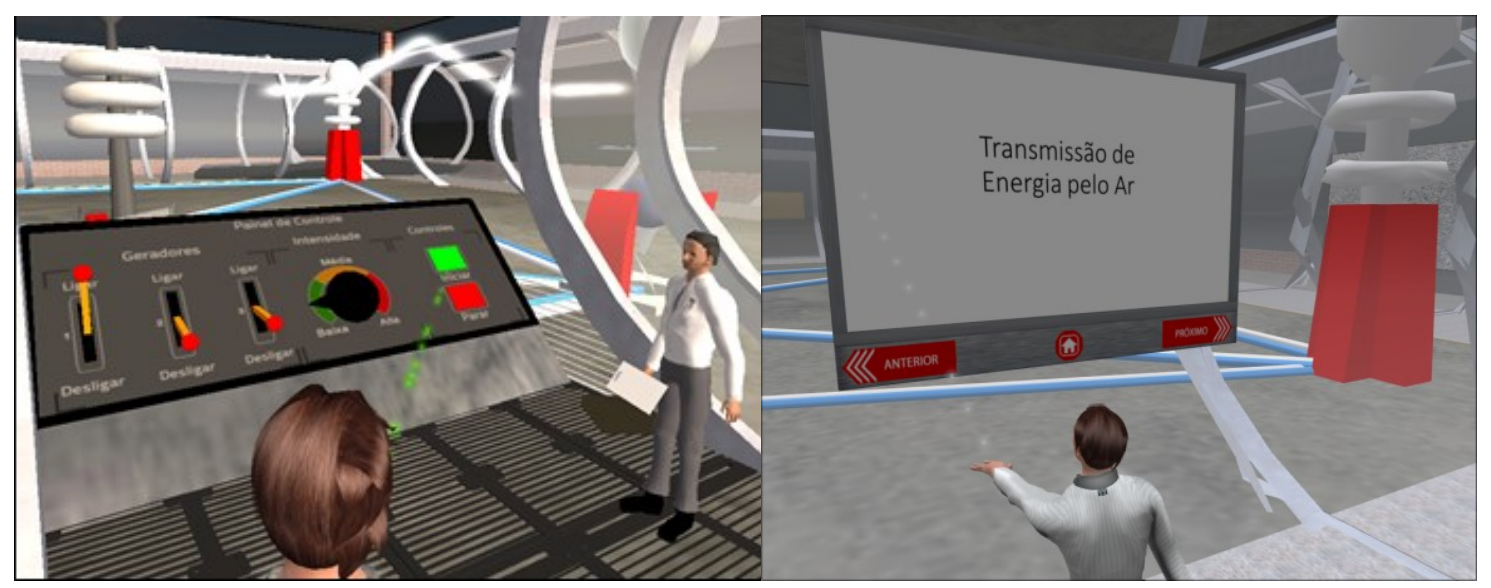

Figura 1. Execução do Experimento

Figura 2. Apresentação de Conceitos

Para o desenvolvimento do laboratório de física, foram utilizadas as funcionalidades nativas do OpenSim para a construção de objetos primitivos, como também foram empregadas ferramentas de autoria, e.g. Blender e SketchUp, para a construção e posterior importação de objetos tridimensionais no laboratório. De forma complementar aos objetos construídos e importados, também foram elaborados scripts 
nas linguagens Linden Scripting Language (LSL) e OpenSimulator Scripting Language (OSSL), aos quais foram atribuídas funções com comportamentos objetivando a execução da simulação da bobina de Tesla, assim como demonstrado na Figura 1.

Além da implementação de scripts com comportamentos a serem executados visualmente aos usuários, também foram construídos scripts que possibilitaram, por meio de funções de sensoriamento, a coleta de dados durante as interações dos usuários com os recursos educacionais disponíveis no laboratório virtual. Estes sensores foram desenvolvidos por meio do uso das linguagens LSL e OSSL, e tinham como objetivo realizar tarefas de requisições externas e respostas, as quais possibilitaram o armazenamento de informações acerca da navegação e interação destes usuários.

No decorrer da próxima seção, serão discutidos os resultados alcançados com a prática da tarefa de associação em mineração de dados e, consequentemente, os padrões e relacionamentos evidenciados no processo de aquisição de conhecimento a partir da análise do conjunto de dados selecionado para esta investigação.

\section{Análise e Discussão dos Resultados}

Efetuada as descrições acerca da forma com que foi conduzido o estudo de caso, esclarecendo o modo de implementação das atividades no mundo virtual, forma de coleta dos dados e a metodologia de análise e mineração dos dados, se torna necessário executar a interpretação dos resultados. Desta forma, esta seção irá apresentar os resultados obtidos com a mineração dos dados e detalhar as interpretações das informações discriminadas.

Com base nos registros coletados pelos sensores, foi possível estabelecer as interações que os usuários realizaram durante a navegação. Sendo que a partir destes registros, os dados foram selecionados, passando por um pré-processamento do conjunto de dados e posterior formatação, até ser desempenhada a mineração de dados propriamente dita. Ao término da execução da tarefa de associação em mineração de dados, as regras evidenciadas foram interpretadas e analisadas junto ao contexto em que fazem parte, com o intuito de avaliar os conhecimentos relevantes para o engajamento dos usuários no laboratório virtual de física.

Para desempenhar a tarefa de associação em mineração de dados, foi utilizado o algoritmo Apriori, o qual possibilita a extração de regras de associação existentes no conjunto de dados selecionados. O algoritmo Apriori é frequentemente utilizado em bancos de dados que armazenam grande volume de informações, sendo que por meio de sua aplicação, deseja-se descobrir as associações importantes entre os itens das relações que compõem este banco de dados, e.g. evidenciar se a presença de alguns destes itens, em uma interação que configure o engajamento do usuário, implica na existência de outros na mesma interação. A partir disso, o Apriori tem como objetivo encontrar um número de regras de associação relevantes entre os itens do conjunto de dados selecionado, as quais são representadas da seguinte forma: $X$ (antecedente) $=>$ (implica em) Y (consequente).

Esclarecido tais conhecimentos pertinentes para a problematização abordada neste artigo, as associações descobertas com a análise dos itens que compõem o banco de dados deste estudo foram: 
V Congresso Brasileiro de Informática na Educação (CBIE 2016)

Anais dos Workshops do V Congresso Brasileiro de Informática na Educação (CBIE 2016)

\section{Quadro 1. Regras de Associação evidenciadas}

\begin{tabular}{|c|}
\hline 02. Experimento=Sim $15==>$ Avaliacao_2=Sim 15<conf:(1)> lift:(1.18) lev:(0.11) [2] conv:(2.25) \\
\hline 03. Experimento=Sim Avaliacao_2=Sim $15=\Rightarrow$ Apresen_1=Sim $15<$ conf:(1)> lift:(1.18) lev:(0.11) [2] conv:(2.25) \\
\hline 04. Experimento $=$ Sim Apresen_1 $=$ Sim $15=>$ Avaliacao_ $2=\operatorname{Sim} 15<$ conf:(1) $>$ lift:(1.18) lev:(0.11) [2] conv:(2.25) \\
\hline 05. Experimento=Sim $15==>$ Apresen_1=Sim Avaliacao_2=Sim 15<conf:(1) $>$ lift:(1.25) lev:(0.15) [2] conv:(3) \\
\hline 06. Apresen_1=Sim Avaliacao_1=Sim $15==>$ Pergunta_1=Sim 15<conf:(1)> lift:(1.18) lev:(0.11) [2] conv:(2.25) \\
\hline 07. Pergunta_1=Sim Avaliacao_2=Sim $15==>$ Apresen_1=Sim 15<conf:(1)> lift:(1.18) lev:(0.11) [2] conv:(2.25) \\
\hline 08. Avaliacao_1=Sim Avaliacao_2=Sim $15==>$ Apresen_1=Sim $15<$ conf:(1)> lift:(1.18) lev:(0.11) [2] conv:(2.25) \\
\hline 09. Apresen_1=Sim Avaliacao_1=Sim $15=\Rightarrow$ Avaliacao_2=Sim 15<conf:(1)> lift:(1.18) lev:(0.11) [2] conv:(2.25) \\
\hline
\end{tabular}

Com relação as associações evidenciadas no conjunto de dados analisado, conforme apresentado no Quadro 1, as regras extraídas transpassam a noção exata dos elementos e recursos educacionais com que os usuários interagiram durante a navegação no laboratório virtual de física. Neste contexto, é possível verificar que os recursos: (a) experimento, (b) apresentações e (c) perguntas, foram frequentemente associados às (d) avaliações, o que fornece fortes indícios de que contribuíram para o usuário desempenhar tais avaliações.

De forma mais específica, devido à grande quantidade de manifestação do recurso experimento nas regras evidenciadas, ou seja nas regras de 1 a 5 , é possível inferir que o mesmo foi um dos fatores que possibilitou o envolvimento dos usuários. Esta constatação pode ser interpretada devido ao alto grau de interação que o recurso possibilitou aos usuários, uma vez que tinham a possibilidade de iniciar e parar a simulação, alterar seus valores de execução, bem como visualizá-la de diferentes ângulos.

Através das regras encontradas neste conjunto de dados e outras informações constatadas, é possível afirmar que na amostragem analisada, os recursos evidenciados que favoreceram o engajamento destes usuários com o laboratório virtual de física foram:

- Experimentos interativos: que possibilitam ao usuário interagir com a simulação, iniciar, parar e alterar seus valores de execução, bem como verificá-los por outros ângulos;

- Conteúdos educacionais simples e objetivos: que promovam a interação do usuário com apresentações, imagens e vídeos, as quais demonstram de forma clara e sucinta o conteúdo que está sendo abordado;

- Frequentes questionamentos sobre os conteúdos apresentados: que instigam o usuário a envolver-se com os tópicos apresentados com o intuito de responder a uma determinada avaliação; 
V Congresso Brasileiro de Informática na Educação (CBIE 2016)

Anais dos Workshops do V Congresso Brasileiro de Informática na Educação (CBIE 2016)

- Avaliações com feedbacks imediatos: para que o usuário possa identificar o que respondeu de forma incorreta e/ou como seria a solução adequada do problema;

- Ganhos do usuário em suas interações: por meio das interações que o usuário realiza com os recursos educacionais, o mesmo consegue identificar os ganhos que estas interações podem oferecer para o seu aprendizado;

- Atividades que contemplem os aspectos colaborativos: que promovem a interação entre os usuários do laboratório virtual, a fim de que os mesmos auxiliem uns aos outros na realização de atividades;

- Comunicabilidade: para que os usuários do laboratório virtual possam trocar mensagens de texto e voz instantâneas, característica que possibilita aos mesmos o intercâmbio das percepções sobre as experiências realizadas, inclusive possibilitando um usuário auxiliar o outro na resolução de alguma atividade.

Diante do que foi descoberto com a geração das regras de associação, a partir de um conjunto de transações com os itens em um banco de dados, possíveis ações podem ser executadas para a melhoria do laboratório virtual de física. Neste sentido, estes conhecimentos podem ser aplicados com o intuito de desenvolver uma sequência mais apropriada aos materiais educacionais, que torne a interação dos usuários mais envolvente. Nesta perspectiva, o conhecimento evidenciado pelas regras de associação pode ser utilizado para desempenhar algumas ações que permitam reorganizar os materiais educacionais aos quais os usuários tem acesso, por exemplo, estabelecendo uma ordem que acompanhe as preferências ressaltadas nas associações.

\section{Conclusão}

A evolução dos recursos tecnológicos na educação permitiu que novas alternativas pudessem ser utilizadas para auxiliar no processo de aprendizagem. Os mundos virtuais estão dentre estas alternativas, no qual a exploração dos aspectos ligados ao engajamento dos usuários nestes ambientes, pode ser considerado um tópico emergente e relevante. Agregado a este escopo, está a aplicação da mineração de dados, que em virtude da grande quantidade de dados provenientes do mundo virtual, se caracteriza necessária a execução de técnicas para a análise dos dados ligados ao engajamento dos usuários.

Com o desenvolvimento desta investigação, foi possível estabelecer a análise dos dados coletados no laboratório virtual de física, por intermédio da execução da tarefa de associação em mineração de dados. Através desta análise, foi possível determinar indícios que demonstram quais são os elementos e recursos educacionais presentes neste laboratório, capazes de promover o engajamento dos usuários e motiválos a retornarem ao mesmo em busca de novas interações.

Outro aspecto evidenciado nas regras de associação descobertas durante a análise realizada neste estudo, consiste na possibilidade de oferecer um panorama geral aos professores que desenvolvem ou utilizam estes ambientes virtuais imersivos em sala de aula, para que os mesmos consigam verificar o que está acontecendo durante o processo de aprendizagem do estudante, visto que os dados coletados podem oferecer um feedback sobre a navegação de cada usuário e suas interações com os elementos educacionais. Em conformidade com isso, também é possível verificar aqueles recursos educacionais que não são importantes no ponto de vista pedagógico. 
Em relação as perspectivas de trabalhos futuros desta investigação, pretende-se continuar a coleta de dados com os usuários do laboratório virtual de física, a fim de obter um volume de dados considerável para uma posterior análise, inclusive utilizando novas tarefas de mineração de dados. Também objetiva-se utilizar os conhecimentos descobertos ao longo deste estudo, para ampliar os meios de engajamento dos usuários, explorando os aspectos que foram ressaltados com as regras de associação, assim como buscando novas formas de envolver os usuários.

\section{Referências Bibliográficas}

Baker, R., Isotani, S., \& Carvalho, A. (2011). Mineração de Dados Educacionais: Oportunidades para o Brasil. Revista Brasileira de Informática na Educação, v.19, n.2.

Castanheira, L. G. (2008). Aplicação de Técnicas de Mineração de Dados em Problemas de Classificação de Padrões. Universidade Federal de Minas Gerais (UFMG).

Chiquito, A. J., \& Lanciotti Jr., F. (2000). Bobina de Tesla: dos Circuitos Ressonantes LC aos Princípios das Telecomunicações. Revista Brasileira de Ensino de Física, v. 22, n. 1, p. 69-77.

Costa, E., Baker, R. S. J. d., Amorim, L., Magalhães, J., \& Marinho, T. (2012). Mineração de Dados Educacionais: Conceitos, Técnicas, Ferramentas e Aplicações. Jornada de Atualização Em Informática Na Educação (JAIE), v. 1, n. 1, p. 1-29.

Fayyad, U., Piatetsky-Shapiro, G., \& Smyth, P. (1996). From data mining to knowledge discovery in databases. AI Magazine, v. 17, n. 3, p. 37-54.

Fernández-Gallego, B., Lama, M., Vidal, J. C., \& Mucientes, M. (2013). Learning Analytics Framework for Educational Virtual Worlds. International Conference on Virtual and Augmented Reality in Education, p. 443-447.

Herpich, F., \& Tarouco, L. M. R. (2016). Engajamento de Usuários em Mundos Virtuais: uma Análise Teórica-Prática. RENOTE, v. 14, n. 1, p. 1-11.

Nunes, F. B., Herpich, F., Voss, G. B., Medina, R. D., Lima, J. V. De, \& Tarouco, L. M. R. (2014). Laboratório Virtual de Química: uma ferramenta de estímulo à prática de exercícios baseada no Mundo Virtual OpenSim. III CBIE - XXV Simpósio Brasileiro de Informática Na Educação (SBIE), p. 712-721.

Nunes, F. B., Voss, G. B., \& Cazella, S. C. (2015). Mineração de dados educacionais e Mundos Virtuais: um estudo exploratório no OpenSim. Anais dos Workshops do IV Congresso Brasileiro de Informática na Educação (CBIE), p. 1044-1053.

O’Brien, B. H. L., \& Toms, E. G. (2008). What is User Engagement? A Conceptual Framework for Defining User Engagement with Technology. Journal of the American Society for Information Science \& Technology, v. 59, n. 6, p. 938-955.

Orgaz, G. B., R-Moreno, M. D., Camacho, D., \& Barrero, D. F. (2012). Clustering avatars behaviours from virtual worlds interactions. Proceedings of the 4th International Workshop on Web Intelligence \& Communities - WI\&C'12. NY, USA: ACM Press.

Romero, C., \& Ventura, S. (2010). Educational Data Mining: A Review of the State of the Art. IEEE Transactions on Systems Man and Cybernetics Part C (Applications and Reviews), v. 40, n. 6, p. 1-18. 